\title{
Whole-exon sequencing of human myeloma cell lines shows mutations related to myeloma patients at relapse with major hits in the DNA regulation and repair pathways
}

Benoît Tessoulin ${ }^{1,2^{*}}$ (D), Agnès Moreau-Aubry ${ }^{1}$, Géraldine Descamps', Patricia Gomez-Bougie', Sophie Maïga', Alban Gaignard ${ }^{3}$, David Chiron ${ }^{1}$, Emmanuelle Ménoret ${ }^{4}$, Steven Le Gouill ${ }^{1,2}$, Philippe Moreau ${ }^{1,2}$, Martine Amiot ${ }^{1}$ and Catherine Pellat-Deceunynck ${ }^{1 *}$

\begin{abstract}
Background: Human myeloma cell lines (HMCLs) are widely used for their representation of primary myeloma cells because they cover patient diversity, although not fully. Their genetic background is mostly undiscovered, and no comprehensive study has ever been conducted in order to reveal those details.

Methods: We performed whole-exon sequencing of $33 \mathrm{HMCLs}$, which were established over the last 50 years in 12 laboratories. Gene expression profiling and drug testing for the $33 \mathrm{HMCLs}$ are also provided and correlated to exonsequencing findings.

Results: Missense mutations were the most frequent hits in genes (92\%). HMCLs harbored between 307 and 916 mutations per sample, with TP53 being the most mutated gene (67\%). Recurrent bi-allelic losses were found in genes involved in cell cycle regulation (RB1, CDKN2C), the NFKB pathway (TRAF3, BIRC2), and the p53 pathway (TP53, CDKN2A). Frequency of mutations/deletions in HMCLs were either similar to that of patients (e.g., DIS3, PRDM1, KRAS) or highly increased (e.g., TP53, CDKN2C, NRAS, PRKD2). MAPK was the most altered pathway (82\% of HMCLs), mainly by RAS mutants. Surprisingly, HMCLs displayed alterations in epigenetic (73\%) and Fanconi anemia (54\%) and few alterations in apoptotic machinery. We further identified mutually exclusive and associated mutations/deletions in genes involved in the MAPK and p53 pathways as well as in chromatin regulator/modifier genes. Finally, by combining the gene expression profile, gene mutation, gene deletion, and drug response, we demonstrated that several targeted drugs overcome or bypass some mutations.

Conclusions: With this work, we retrieved genomic alterations of HMCLs, highlighting that they display numerous and unprecedented abnormalities, especially in DNA regulation and repair pathways. Furthermore, we demonstrate that HMCLs are a reliable model for drug screening for refractory patients at diagnosis or at relapse.
\end{abstract}

Keywords: Myelomas, WES, Tumor suppressors, DNA repair, Fanconi pathway

\footnotetext{
* Correspondence: benoit.tessoulin@chu-nantes.fr; catherine.pellatdeceunynck@univ-nantes.fr

${ }^{1}$ CRCINA, INSERM, CNRS, Université d'Angers, Université de Nantes, Nantes,

France

Full list of author information is available at the end of the article
}

(c) The Author(s). 2018 Open Access This article is distributed under the terms of the Creative Commons Attribution 4.0 International License (http://creativecommons.org/licenses/by/4.0/), which permits unrestricted use, distribution, and reproduction in any medium, provided you give appropriate credit to the original author(s) and the source, provide a link to the Creative Commons license, and indicate if changes were made. The Creative Commons Public Domain Dedication waiver (http://creativecommons.org/publicdomain/zero/1.0/) applies to the data made available in this article, unless otherwise stated. 


\section{Background}

Human myeloma cell lines (HMCLs) are widely used for their representation of primary myeloma cells because they cover patient diversity, although not fully [1]. HMCLs are mainly derived from refractory patients, mostly presenting with extramedullary disease and having thus received numerous classes of drugs inducing DNA damage, proteasome inhibition, immunomodulation, and anti-inflammation (e.g., melphalan, bendamustine, Velcade, Revlimid, and dexamethasone). However, HMCLs harbor the 14q32 abnormality, which occurs early at the MGUS stage, and display frequent mutations in NRAS and KRAS, as observed in patients at diagnosis (approximately $50 \%$ of patients) [2, 3]. By contrast, HMCLs display very frequent deletion and mutation in the TP53 gene that are associated with resistance to treatments [4]. Indeed, it is well known that hits in the TP53 gene (deletion and/or mutation) at diagnosis are associated with resistance and shortened survival and that their frequency increases with relapse $[4,5]$. Thus, HMCLs are a mixture of abnormalities occurring both early and late in the time course of disease. Besides hits in the TP53 and RAS genes, HMCLs have not been widely characterized for their global mutation profile and gene deletion. In the present work, using wholeexon sequencing (WES) in 33 HMCLs, we report common gene mutations and deletions. We analyzed the frequency of mutations/deletions in comparison with patients at diagnosis and relapse. We further identified hits preferentially associated with $14 \mathrm{q} 32$ translocations and analyzed responses to conventional and nonconventional drugs in relation to a mutation and/or deletion profile.

\section{Methods}

\section{HMCLs and primary MM cells}

HMCLs were previously characterized [1, 6, 7]. HMCLs were cultured in RPMI-5\% fetal calf serum with or without $3 \mathrm{ng} / \mathrm{ml}$ of IL6 [1, 6, 7]. Gene expression profile of HMCLs has been previously published [1]. The gene expression profile of primary MM cells was assessed from 414 patients (Arkansas) as previously described [1, 8].

\section{Whole-exon sequencing}

DNA sample processing was performed according to Agilent Technologies (Santa Clara, CA, USA) using the sureselect target enrichment system kit (Human all exon v6, library version 1.6). Sequencing was performed on HiSeq 2500, High Output in paired-end $2 \times 100 \mathrm{bp}$. The reads were aligned (BWA-v0.7.10-r789) to the GRCh37 human reference genome. Duplicated reads were marked by the Picard tool (v1.119), indels were realigned around capture $( \pm 500 \mathrm{bp})$, and base quality recalibration was finally performed (Genome Analysis Toolkit [GATK-v3.2.2]). In the absence of germline DNA, variants were called by the GATK unified genotyper. Variants were processed through vcf2maf-1.6.15 to obtain a final Mutant Annotation File (maf). The variants' biological effects predictions were carried out using Ensembl's VEP-annotator-v.86. Variant annotation database versions were as follows: ExAC-r0.3.1 [likely germline variants], dbSNP-v.144 [known variants], COSMIC-71 and ClinVar-v.201507 [clinical significance of known variants].

Variants that were present more than three times were removed, as well as variants with Global Allele Frequency in ExAC databases over 1\% (with respect to ethnicity frequencies when known). Finally, clinically benign mutants, as annotated by ClinVar, were removed ("benign" or "likely benign"). Only protein-coding variants were used for subsequent analyses, and structural protein coding genes (actin, myosin, collagen, fibronectin, vitronectin, tenascin, laminin, titin, obscurin, plectin, aggrecan, and mucins) were removed.

Exon loss was estimated from the read depth using ExomeCOPY and CANOES. The results were validated by visual inspection of the BAM read depth in Integrative Genomics Viewer (IGV; Broad Institute). Genes with frequent variants were selected and were assessed by direct Sanger sequencing on cDNA.

\section{Functional assays}

The cell count and viability were measured using the MTT assay. The cell cycle distribution was assessed by propidium iodide incorporation. $\mathrm{Rb}$ phosphorylation was assessed by western blotting (Cell Signaling; 4H1 and S807-811). The area under the curve (AUC) was estimated using Graphpad Prism v7.0 for palbociclib $(0-1 \mu \mathrm{M})$, CX5461 (0-1 $\mu \mathrm{M})$, and trametinib $(0-25 \mathrm{nM})$. The responses to melphalan, bendamustine, FAS and TRAIL-R agonist antibodies, PRIMA-1 ${ }^{\text {Met }}$, dexamethasone, RITA, ABT-737, and ABT-199 were previously reported [6, 9-14]. Results were scaled (mean-centered and standardized) to provide a $z$-score.

\section{Statistical analyses}

Analyses were performed under R 3.4.4. Fisher's test was carried out with the resampling of parameters for robustness. The somatic interaction plot code was adapted from Gerstung et al. [15]. Enrichment analyses were carried out by ReactomePA and clusterProfiler [16, 17], $p$ values were adjusted for multiple testing by the false discovery rate $(q=0.05)$. For the Reactome determination, KEGG and GO annotations were used. MAF manipulation was performed using the maftools packages [18]. Oncoprints, heatmaps, and Chord-Diagrams were performed with ComplexHeatmap R-package. Considering the number of samples, the linear regressions between scores and drug responses were calculated by robust a linear regression using a M-estimator (rlm, MASS 
package) in order to discard outliers. Coefficients were further bootstrapped by Boot function (car package), with 5000 replicates (seed $=22,062,016)$ and considered significant if the $95 \%$ confidence interval $(95 \% \mathrm{CI})$ did not overlap with zero, only $\beta 1$ coefficients are presented in the text.

\section{Results}

\section{Metrics and variant filtering}

WES was performed in 33 HMCLs of European, American or Asian origin, 19 having been derived in the presence of exogenous IL6 (Additional file 1: Table S1). After global SNP enrichment analysis on 609,585 bi-allelic SNPs (SNPRelate package [19]), three groups of HMCLs were identified: a group gathering HMCLs of Pacific/Japanese origin (AMO-1, KMM1, KMS12PE, KMS11, NAN8, OPM2) and a cluster encompassing all other HMCLs except MM1S, which was individualized as African ethnicity (Additional file 1: Figure S1). To remove ethnic-related SNPs, HMCLs were filtered with Global Allele Frequencies, plus East Asian frequencies for the Pacific/Japanese cluster and African frequencies for MM1S. Because of the lack of normal DNA from patients from whom the HMCLs were derived, we could not easily discriminate the constitutive SNPs from the tumor-associated mutations. Thus, we excluded variants shared by more than 3 HMCLs of the 33: indeed, the most mutated genes in HMCLs and myeloma patients [20], i.e., RAS and TP53, never displayed more than three identical variants across the HMCL collection. For $N R A S$, the most frequent variant was c.38G-A (Gly12Asp) in JJN-3, Karpas620, and Nan7, while the only TP53 shared variant was 406G-A (Karpas620, XG11). Variant effect predictions were carried out as described in the "Methods" section. We further removed variants of genes uniformly low expressed across the collection (maximum of the considered gene inferior to the first quartile mean expression of the microarray). After filtering, we retained 15,602 variants, spanning over 7641 genes (Maf file, Additional file 2). Most mutated samples were KMM1 and KMS12PE with 916 and 755 variants, respectively. The most frequent variant was missense ( $n=14,309 ; 92 \%)$, while frameshifts occurred in 273 variants $(1.7 \%)$, insertions or deletions without frameshifts occurred in 226 cases (1.4\%), and 482 variants (3.1\%) were nonsense mutations (Additional file 1: Figure S2). Single mutations were mainly $\mathrm{C}>\mathrm{T}$ transitions (63\%, Additional file 1: Figure S3), corresponding to spontaneous deamination of 5-methyl cytosine. HMCLs age was not associated with a particular mutation (Fisher test, FDR $>0.05$ ), but younger cell lines displayed a lower mutation load $(\beta=4.29,95 \% \mathrm{CI}=[1.07$; 9.47]). Mutations were confirmed in 18 genes by direct sequencing of RT-PCR products as previously reported for RAS and TP53 [1, 9] (Additional file 1: Table S1). Although amplification of genes was not assessed because of the high number of chromosome abnormalities across the HMCL collection, exon losses were reported as described in the "Methods" section. Main variants are presented in Lollipop Plots (Additional file 3).

\section{HMCLs display alterations similar to those in MM cells}

Figure 1 shows the most frequently altered genes across the collection and recurrent in MM [20-22]. Residues modified by mutants are provided in Additional file 1: Figure S4. Five genes, i.e., TP53, KRAS, NRAS, CDKN2C and PRKD2 were altered in at least $21 \%$ and up to $67 \%$ of HMCLs.

HMCLs shared a similar mutations rate with MM patients, either at diagnosis (DMM) or relapse (RMM) for DIS3 (12\% in HMCLs, 10\% in DMM and 13\% in RMM), PRDM1 (3\%, 2\%, and 5\%, respectively), BIRC3 (3\%, 2\%, and $3 \%$, respectively), and $E G R 1$ (3\%, $4 \%$, and $4 \%$, respectively) (Fig. 2). Of note, these very similar rates among HMCLs, DMM and RMM were in favor of early pathogenic mutations, poorly affected by subsequent treatment selection or cell culture. KRAS mutation rates were roughly shared between HMCLs, DMM, and RMM (21\%, $24.7 \%$, and $27 \%$, respectively). By contrast, the NRAS mutation rate increased from DMM (19\%) to RMM (24\%) and HMCLs (30\%). Similarly, TP53 (67\%), CDKN2C (33\%), PRKD2 (18\%), FAM46C (15\%), and $B R A F(15 \%)$ mutation rates displayed a dramatically increased frequency in HMCLs compared with those in primary myeloma cells, either in DMM or RMM [2, 20-24] (Fig. 2). These high frequencies in the FAM46C, TP53, BRAF, and NRAS rates might be in line with either successive relapses and/or secondary plasma cell leukemia (PCL), from which HMCLs are mostly derived [25-29].

WES revealed that HMCLs displayed frequent mutations in Fanconi anemia genes (PALB2 [12\%], FANCI [12\%], FANCA [9\%], FANCD2 [9\%], BRCA2 [9\%]) as well as in helicases (such as RECQL4, 15\%, and BLM, $15 \%$ ) and epigenetic modifiers (e.g., TET2, 15\% and SETD2, 6\%). FANC family genes were recently reported to be mutated mostly in patients at relapse [24, 30, 31], suggesting that these mutations did not occur in vitro in continuously replicating cells but in vivo. Mutations in epigenetic modifiers were recently described as being more frequent at relapse [20, 22, 24, 32], such as histone methyl-transferases $(6.9 \%$ vs $17 \%$, in DMM and RMM, respectively) and DNA methylation modifiers (1.9\% vs $8.3 \%$, respectively). On the other hand, genes involved in apoptotic pathways (extrinsic, intrinsic, execution) displayed few mutations or deletions, showing that cell death resistance was not associated with major defects in the apoptotic machinery. 


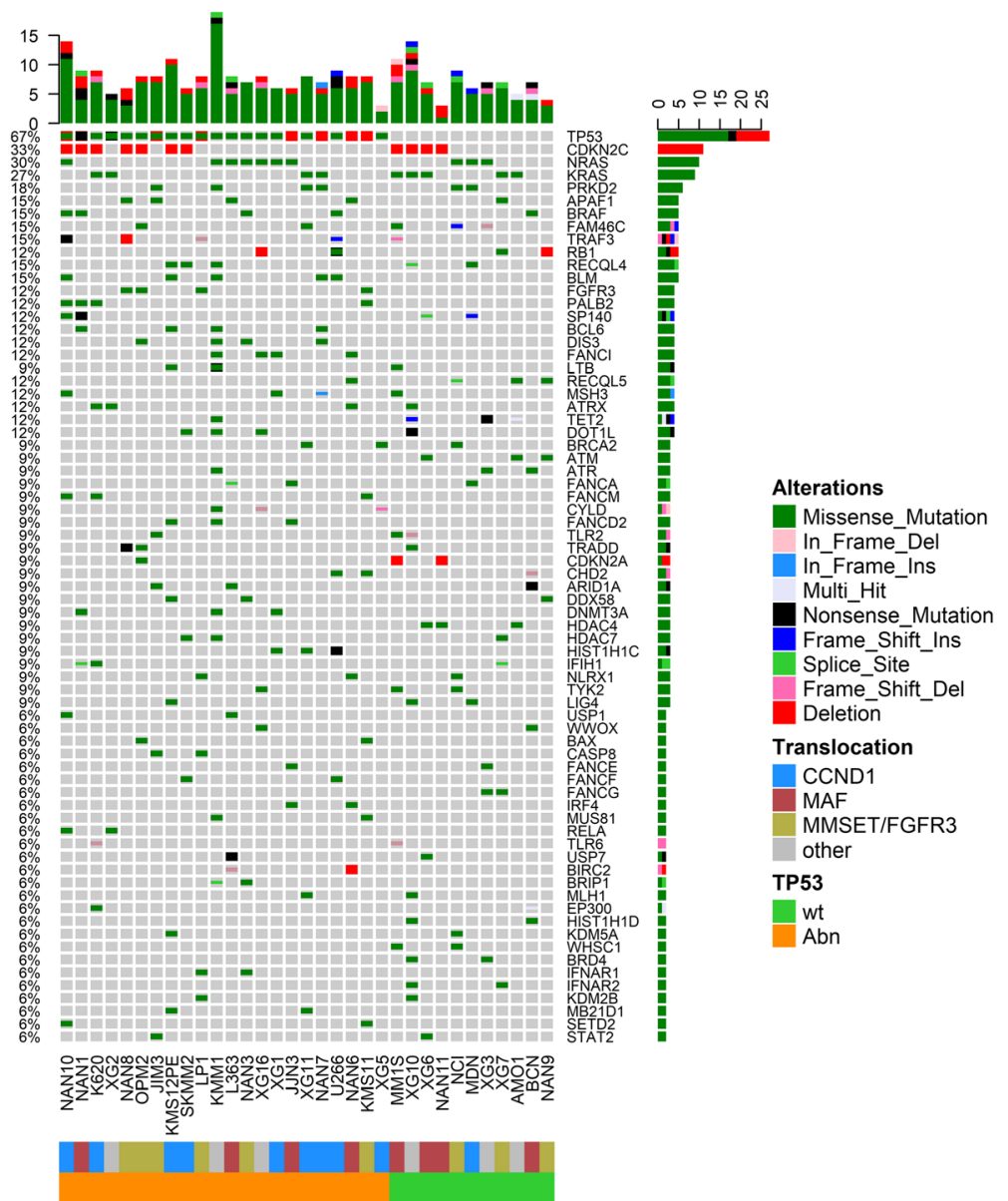

Fig. 1 Oncoprint of the most frequently mutated and/or deleted genes in human myeloma cell lines. HMCLs were ranked according to the most frequent abnormalities. Several events affecting the same cell line (mutations and deletion) were represented in the same slot. The number of cumulative events per $\mathrm{HMCL}$ is indicated on the top of the graph

These data collectively showed that HMCLs displayed mutations/deletions related to myeloma cells from patients at diagnosis (KRAS, DIS3, EGR1, PRDM1 and $B I R C 3)$ and relapse/progression to PCL (TP53, NRAS, $B R A F, F A N C$ genes).

We further analyzed mutually exclusive and co-occurring mutations/deletions, as well as their associations with IgH translocation (Additional file 1: Figure S5). Abnormalities in the Ras/MAPK pathway were mutually exclusive: KRAS, NRAS, HRAS, FGFR3 (and to a lesser extent $B R A F)$ displayed mutually exclusive mutations ( $p$ $<0.05$ ), and FGFR3 mutations were exclusively found in $\mathrm{t}(4 ; 14)$ HMCLs overexpressing FGFR3 $(p=0.003$, Additional file 1: Figure S5). TP53 hits were mutually exclusive to mutations in ATM $(p=0.03)$, as previously reported in all B cell malignancies [33]. By contrast, several co-occurring mutations were found in DNA damage/repair/epigenetic modifiers for instance, in $B L M$ and FANCD2, RECQL5, and ATM, HDAC7, and DOT1L ( $p<$ 0.05). CDKN2A deletion was found in HMCLs with
CDKN2C mutations/deletions. RECQL4 and BLM mutations were significantly associated with $\mathrm{t}(11 ; 14), p=0.03$ and $p=0.002$, respectively.

To provide a comprehensive landscape of mutations/ deletions, we next performed global analysis of altered pathways based on the whole data of the mutated genes.

\section{HMCLs harbor the signatures of dysregulation in Rho GTPase, the cell cycle, and DNA replication}

Gene Ontology (GO) enrichment analysis showed that most of the dysregulated biological processes were related to Rho GTPase signal transduction, Cell cycle/DNA replication and DNA damage (check-points before replication, DNA repair, DNA unwinding) (Additional file 1: Figure S6A). GO molecular functions such as helicase activity, nuclease activity, and Rho GTPase activity were also highly enriched (Additional file 1: Figure S6B-C).

Reactome Pathway Enrichment analysis revealed oncogenic MAPK signaling. After relaxing the $q$ value at 0.1 , pathways involved in DNA repair, p53 regulation of 


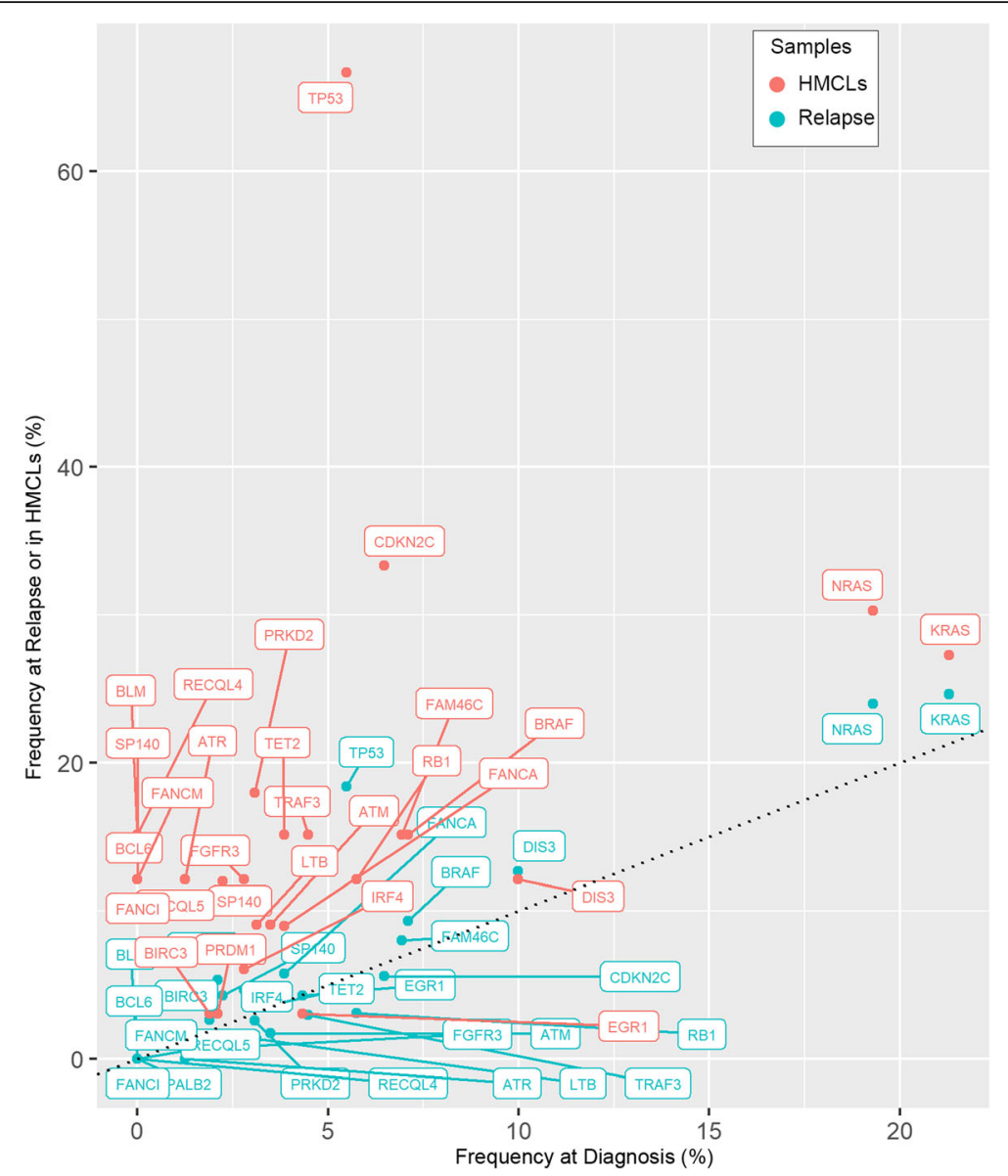

Fig. 2 Comparison of the gene mutation/deletion frequency in human myeloma cell lines with multiple myeloma patients at diagnosis and relapse. The frequency of mutation/deletion at diagnosis ( $x$-axis) was plotted against that at relapse (y-axis, blue). HMCL hit frequencies are represented in red dots. The dashed line represents the theoretical identical ratio between diagnosis and relapse

activity, and DNA double helix-modifying pathways were highlighted, as well as defects in the SUMOylation of DNA replication proteins, DNA damage response and repair proteins, cell cycle regulation by $\mathrm{p} 53$, resolution of D-loop structures through Holliday junction intermediates, and DNA repair (Additional file 1: Figure S6D).

Among cancer-associated pathways, the main KEGGenriched pathways were the Fanconi anemia pathway ( $q$ value $=0.006)$, cell cycle $(q=0.01)$, prostate cancer $(q=$ $0.02)$, chronic myeloid leukemia $(q=0.03)$, non-small cell lung cancer $(q=0.02)$, bladder cancer $(q$ value $=$ $0.02)$, and hepatocellular carcinoma $(q=0.02)$ (Fig. 6e). Non-homologous end joining $(q=0.04)$, platinum resistance $(q=0.04)$, base-excision-repair $(q=0.04)$, and mismatch-repair $(q=0.04)$ were also enriched in KEGG pathways. The prostate cancer, bladder cancer, nonsmall lung cancer, and non-cancer-related pathways revealed by KEGG enrichment analysis were mostly due to the high RAS/BRAF mutation rates. The hepatocellular carcinoma signature was also enriched by Wnt signaling mutations, while breast cancer signature displayed Notch, Wnt, and PI3K altered signaling.

Additional file 1: Figure S7 summarizes hits in the most dysregulated pathways. While pathway dysregulations were globally well balanced among the recurrent translocation subgroups, genes encoding helicases were more frequently encountered in $\mathrm{t}(11 ; 14)$ cell lines $(p=$ 0.006). On the other hand, intrinsic apoptosis mutants were more frequent in $\mathrm{t}(4 ; 14), 66 \%$ vs $12 \%$ in non $\mathrm{t}(4 ; 14)$ cell lines $(p=0.004)$.

The extrinsic, intrinsic, and executive pathways of apoptosis are mostly unaltered in HMCLs

Thirteen HMCLs displayed one or several mutations in the apoptotic pathway (extrinsic, intrinsic, and executive), which were heterozygous (except in LP1 that displayed a bi-allelic BCL2L11/BIM deletion) (Fig. 3). To assess the impact of mutations, we analyzed the cell death response through either the extrinsic, i.e., response to Fas/Trail-R agonist receptors ( $\mathrm{CH} 11$, mapatumumab, or lexatumumab [9]) or the intrinsic pathway of 


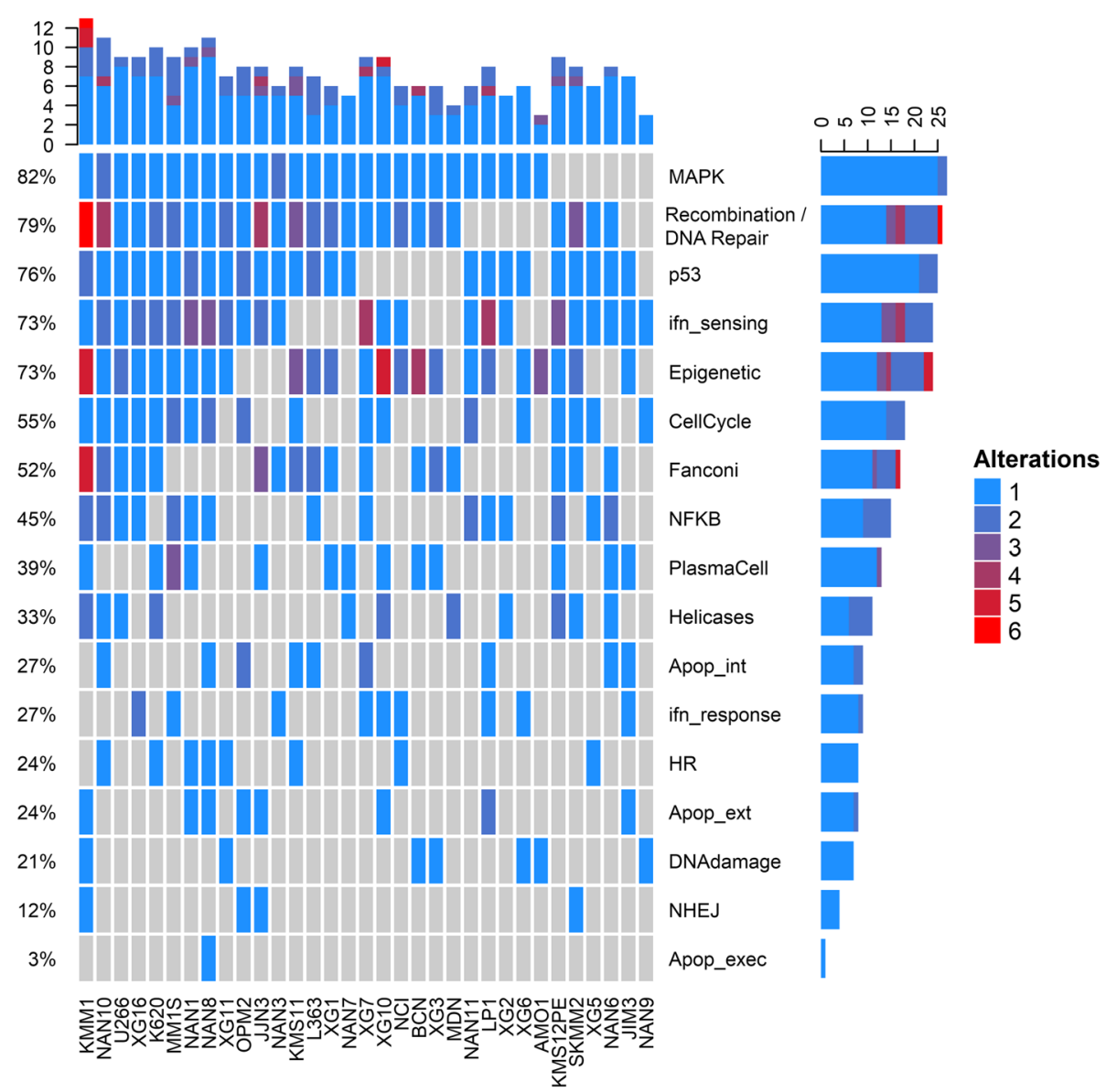

Fig. 3 Oncoprint of altered pathways in human myeloma cell lines. Oncoprint of frequently altered pathways was performed as described in the "Methods" section. Oncoprint was performed with ComplexHeatmap R-package

apoptosis, i.e., response to BH3-mimetics (ABT-199, A-1210477, or A-1155463) [10, 11, 34, 35] (Additional file 1: Table S2). Pathway hit scores were calculated according to the number of hits in each pathway. No correlation could be drawn between the sensitivity to Trail-R agonists or Fas ligands and extrinsic apoptosis hits. Similarly, ABT-199/-737 responses did not correlate with intrinsic apoptosis hits. Moreover, intrinsic pathway hits were not associated with BH3-profiling, i.e., cytochrome $\mathrm{C}$ release in response to BIM peptide (Additional file 1: Table S2), confirming that the heterozygous mutations did not affect the upstream apoptotic responses (Additional file 1: Figure S8).

HMCLs with RAS mutation are highly sensitive to trametinib Eighty-two percent of HMCLs displayed at least one variant of the MAPK pathway, with $60 \%$ of HMCLs bearing a $K-/ H-/ N-R A S$ variant (Fig. 3 and Additional file 1: Figure S9). FGFR3 mutations were present in $12 \%$ of HMCLs but in $50 \%$ of $\mathrm{t}(4 ; 14)$ HMCLs with FGFR3 overexpression (KMS11, LP1, OMP2). Five HMCLs expressed a BRAF mutation with $\mathrm{BCN}$ displaying six non-silent mutations in the PKc-like domain (without evidence of a frameshift). $B R A F$ mutations mostly occurred in the PKc-like domain (four of five mutants), outside of the V600 codon. Two samples displayed both NRAS and BRAF mutations (NAN10 and NAN3). As shown in Fig. 4a, RAS-mutated HMCLs displayed hypersensitivity to the MEK-1/2 inhibitor trametinib (Mann-Whitney test, $p=0.002$ ), while the three FGFR3-mutated HMCLs did not display significant sensitivity. Two HMCLs with BRAF mutations (without concomitant $R A S$ mutation) out of three displayed hypersensitivity to trametinib. Of note, the two $B R A F^{\text {mut }}$ HMCLs displaying high sensitivity to trametinib had variants around V600. Finally, the MAPK pathway hit score was associated with an increased sensitivity to trametinib $\left(\beta_{1}=-1.17,95 \% \mathrm{CI}=[-1.41 ;-0.61]\right)$, which was mainly related to $R A S$ mutations (Fig. $4 \mathrm{~b}$ ).

\section{Abnormalities in cell cycle genes do not favor the response to $\mathrm{CDK}$ inhibition}

Approximately half (55\%) of HMCLs showed impaired cell cycle regulation, mostly bi-allelic deletion of the CDKN2C locus (33\% of HMCLs), RB1 alterations (12\%) 

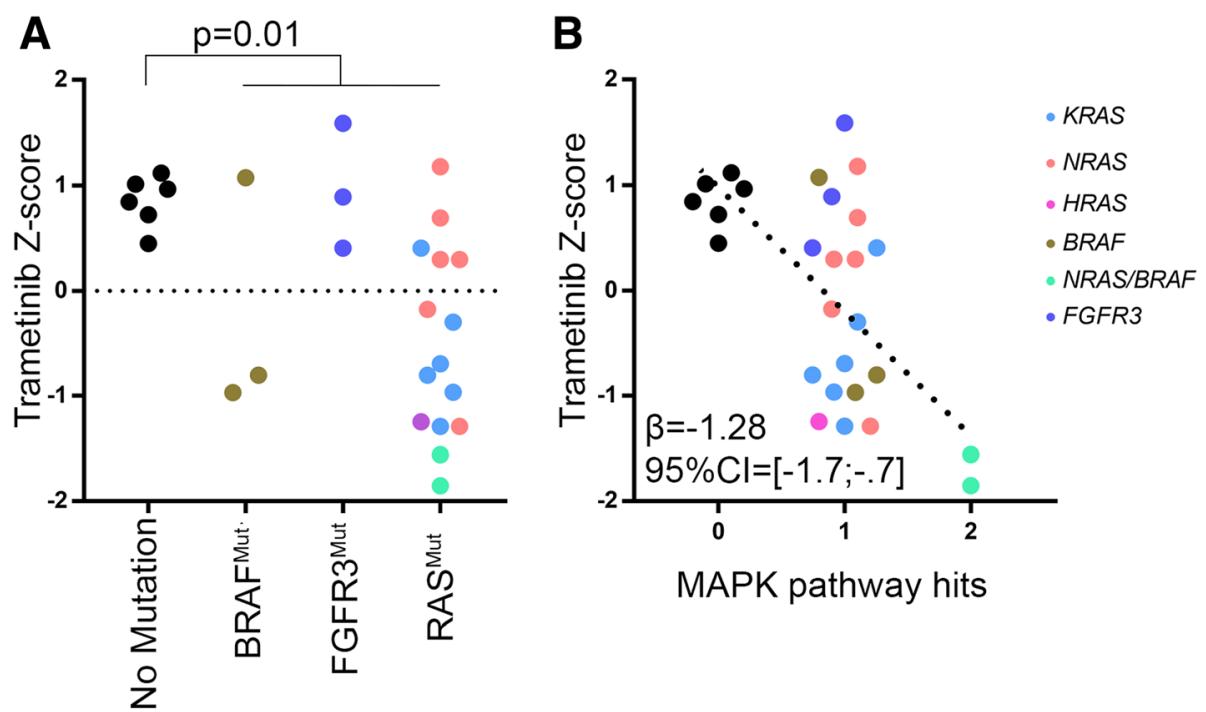

Fig. 4 The sensitivity to trametinib is associated with RAS mutation. a Cells were cultured for 4 days with increasing concentrations of trametinib, and the sensitivity was determined by the area under the curve using the MTT assay and expressed as $z$-score. Analysis was performed as a function of mutations in the MAPK pathway (Mann-Whitney test). b Trametinib response associated with dysregulation in the MAPK pathway. Robust linear regression is displayed; regression line was drawn according to coefficients obtained after 5000 bootstrapped replicates. Points were jittered for clarity

or CDKN2A alterations (9\%) (Additional file 1: Figures S3 \& S5-"CellCycle"). Because deletions in CDKN2A or $C D K N 2 C$, by contrast to $R B 1$ deletion/mutation, were reported to favor cell cycle inhibition by the CDK4/CDK6 inhibitor palbocicib, we assessed whether altered HMCLs were sensitive to the inhibitor [36]. Palbociclib induced an inhibition of cell cycle that was correlated to pRb inhibition $(\beta=-25,95 \% \mathrm{CI}=[-33.8 ;-2.9])$ (Fig. 5a-c). However, no correlation could be found between palbociclib sensitivity and the CDKN2C $(p=0.17), C D K N 2 A(p$ $=0.47)$ or RB1 $(p=0.33)$ status (Mann-Whitney test, Fig. 5d). HMCLs overexpressing CCND1 with an unaltered $R B 1$ showed a trend for better sensitivity than that of other HMCLs ( $p=0.1$ ) (Fig. 5e). Conversely, no correlation was found between palbociclib sensitivity and either the cell cycle pathway score $(\beta=-0.123,95 \% \mathrm{CI}=[-0.89$; $0.53])$ or $C D K 4 / C D K 6$ expression levels $(\beta=0.49,95 \% \mathrm{CI}$ $=[-1.47 ; 1.97]$ and $\beta=-0.02,95 \% \mathrm{CI}=[-0.46 ; 0.5])$, respectively).

\section{Overactivation of NFKB by genomic alterations does not confer oversensitivity to proteasome inhibitors}

The NFkB pathway was altered in $45 \%$ of HMCLs, mostly by inactivating TRAF3 (frame-shift, non-sense mutation, insertion or deletion) or BIRC2/BIRC3 (homozygous deletion) as previously reported in primary myeloma cells $[20,23]$. Alterations in the $N F \kappa B$ pathway were associated with the overexpression of $N F \kappa B$ signature genes [37] (i.e., CD74, TNFAIP3, IL2RG, BIRC3, and $P L E K)$, and we further identified that NFE2L3 (a downstream target of TNF- $\alpha$ signaling displaying a NFKB site in the promoter) was highly expressed in samples harboring NFKB pathway hits (Fig. 6). Furthermore, we found no correlations between $\mathrm{NF} \kappa \mathrm{B}$ pathway dysregulation and sensitivity to proteasome inhibitors $(\beta=0.2,95 \% \mathrm{CI}=[-$ $0.25 ; 0.65]$ for bortezomib and $\beta=-0.09,95 \% \mathrm{CI}=[-0.5$; 0.16] for carfilzomib, Additional file 1: Figure S8).

\section{p53 and DNA damage pathways are associated with} shifts in response to myeloma alkylating drugs

We previously reported that the sensitivity to alkylating drugs was impaired by p53 deficiency [12]. We further assessed whether hits in pathway(s) were associated with the response to drugs reported to be related to p53 deficiency. As shown in Fig. 7, p53 pathway alterations were associated with a lesser response to melphalan $(\beta=0.58$, $95 \% \mathrm{CI}=[0.08 ;-0.9])$ and bendamustine $(\beta=0.63,95 \%$ $\mathrm{CI}=[0.1 ;-1])$. Of note, HMCLs harboring a high DNA damage kinase sensor score had high sensitivity to melphalan $(\beta=-0.83,95 \% \mathrm{CI}=[-1.38 ;-0.27])$ and bendamustine $(\beta=-0.93,95 \% \mathrm{CI}=[-1.52 ;-0.28])$, which was related to TP53 status.

On the other hand, hits in the p53 pathway were also associated with reduced sensitivity to lexatumumab $(\beta=0.71,95 \% \mathrm{CI}=[0.01 ; 1.95])$ and with a trend for increased sensitivity to mapatumumab $(\beta=-0.79$, $95 \% \mathrm{CI}=[-1.24 ;-0.08])$. These correlations were related to the direct and indirect p53-mediated regulation of TNFRSF1OB and TNFRSF10A expression, as previously reported [9]. 


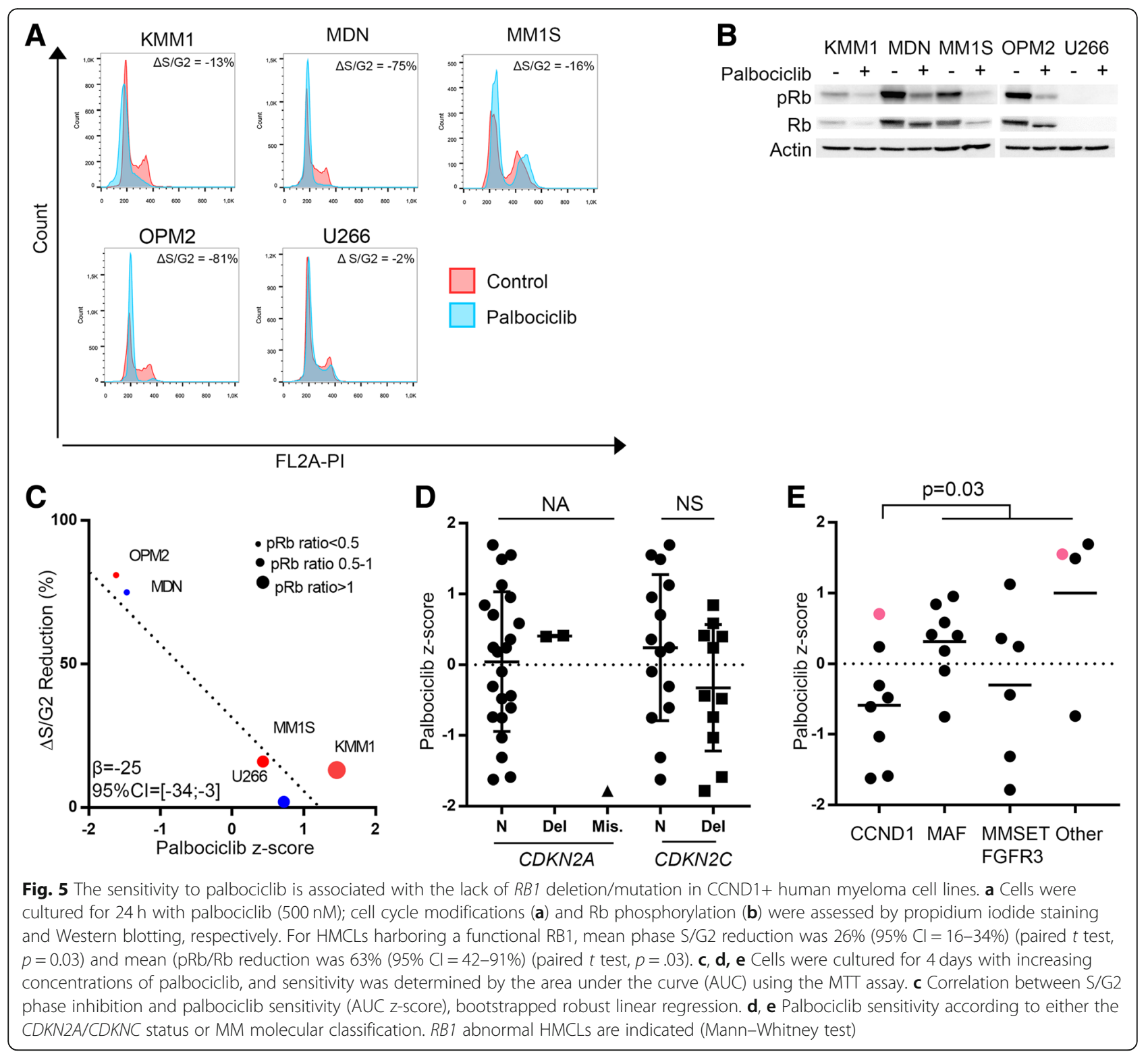

Correlations between other clinically commonly used drugs (Imids, dexamethasone, and proteasome inhibitors) and pathways were not significant (Additional file 1: Figure S8).

\section{Deficiency in the DNA repair pathway does not predict the responses to RITA or CX-5461}

We assessed whether efficacy of DNA targeting drugs could be related to specific alterations. We analyzed sensitivity to RITA and CX-5461, which induce DNA crosslinking and stabilize DNA G-quadruplex, respectively, and are known to involve DNA repair during DNA replication $[13,38,39]$. Since p53 is involved in DNA repair, we analyzed drug responses according to TP53 $3^{\text {status }}$ of HMCLs. Sensitivity to RITA in TP53 ${ }^{w t}$ HMCLs was enhanced by mutations in helicases $(\beta=-0.59,95 \%$ CI $=[-1.04 ;-0.01])($ Fig. 7$)$. HMCL sensitivity to CX-5461 was not associated with any genes or pathway alterations (Additional file 1: Figure S8) although XG11, which displayed a homologous BRCA2 mutation, showed the highest sensitivity to CX-5461.

\section{Discussion}

WES was performed in 33 HMCLs, including 19 that had not been reported yet. HMCLs selected in this study were established between 1965 and 2015 in Europe, USA, or Japan and in the presence or absence of added recombinant IL6. While HMCLs' ages spanned from 55 to 3 years old, mutation load was similar among those, even if more recent HMCLs display lower 

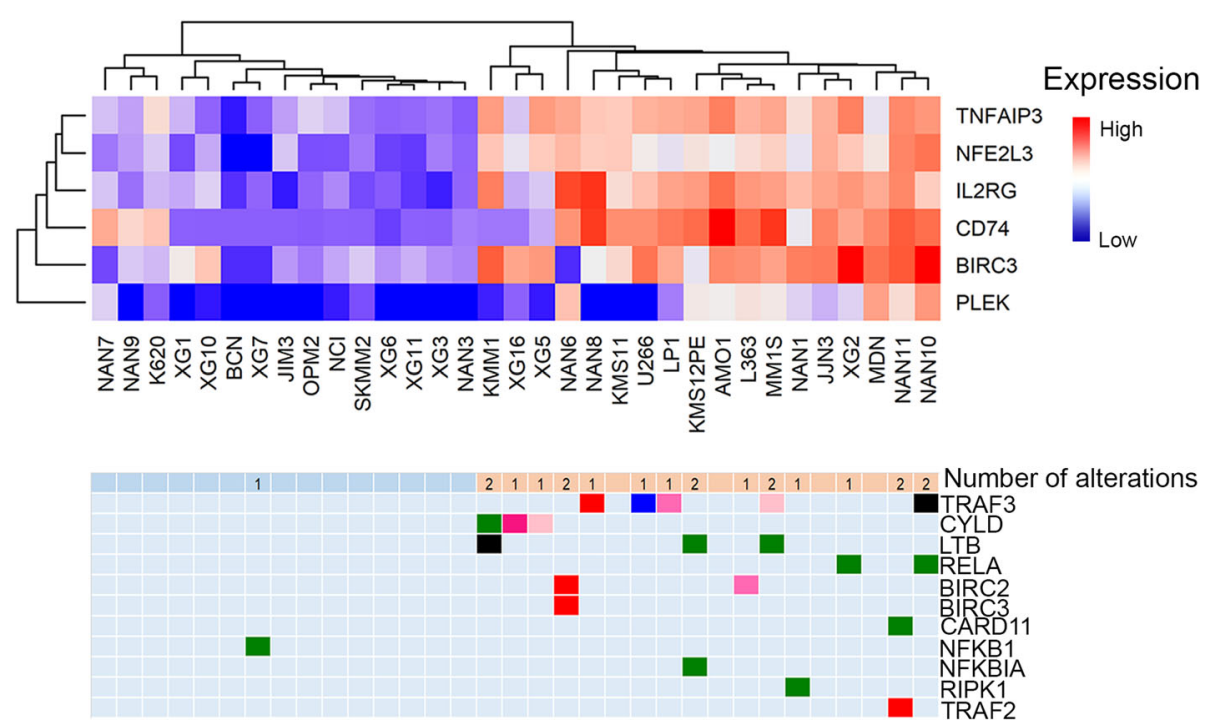

Fig. 6 Mutations/deletions in the NFKB pathway genes are correlated with the overexpression of NFKB target genes. a The expression of genes significantly associated with mutation/deletion in NFkB pathway genes was identified using the limma algorithm. Clustering was performed with the most significant genes. b Representation of the NFKB pathway hits according to HMCL classification in a

mutation load. Our analysis showed that mutated genes were shared between HMCLs and primary myeloma cells, whatever the organ origin of samples that gave rise to the cell lines. Although HMCLs always emerged from patients with extra-medullary disease, no strong comparison could be made with primary or secondary PCL because of the very low number of sequenced PCLs yet, except for del17p (46\% in sPCL) [29]. We thus compared mutation frequency with primary cells at diagnosis and at relapse (without any indication of medullary or extramedullary disease). Although the number of HMCLs was low as compared with patients, we nevertheless identified genes with a very high tumor load suggesting that some of them are drivers. The HMCLs mutational landscape may thus provide a panorama of mutations in refractory patients. The frequency of mutated "myeloma" genes in HMCLs was identical, lower, or higher when compared to primary cells at diagnosis or relapse [20-22]. While mutation rate in KRAS was similar between HMCLs and primary myeloma cells, the frequencies of TP53 (67\%), CDKN2C (33\%), PRKD2 (21\%), FAM46C (15\%), and BRAF $(15 \%)$ dramatically increased compared to primary myeloma cells, either in DMM or RMM. The high TP53 abnormality frequency $(67 \%)$ in HMCLs identified by WES in our study (and confirmed by direct RT-PCR sequencing [1]) was not in good agreement with a previous WES study reporting a rate of $21 \%$ in HMCLs [40], which was highly underestimated: indeed, well-known TP53 mutations in L-363, LP-1, and SKMM-2 (COSMIC database and p53.iarc.fr, Release $=18$ ) were not reported in this study and at least three "HMCLs" were not of myeloma origin (ARH77, MC-CAR, CTV-1) [41, 42].
Our results clearly confirmed a major alteration in both proliferation control, with either loss of suppressor (TP53, CDKN2C, RB1) or acquisition of activator (BRAF, $R A S)$ and in tumor suppression/drug response (TP53, $F A M 46 C$ ), as in most if not all cancers [43]. Because the loss of function of TP53, FAM46C, or CDKN2C are not directly targetable, drugs bypassing these proteins or exploiting their loss consequences are required. Indeed, as shown in Fig. 5, cells lacking CDKN2C expression were sensitive to the CDK4/6 inhibitor palbociclib, especially in the CCND1 group. This CCND1 impact was surprising because palbociclib is efficient against all CDK-CCND complexes, i.e., CDK4/CCND1, CDK4/ CCND3, and CDK6/CCND2 [44]. Of note, CCND2 myeloma cells overexpress $C D K 6$ and $C D K 4$ while $C C N D 1$ myeloma cells overexpress $C D K 4$ but not $C D K 6$, suggesting that CDK4 is "empty" of cyclin D in CCND2 myeloma cells (Additional file 1: Figure S10). This free CDK4 pool might explain the low efficiency of palbociclib in CCND2 HMCLs. Palbociclib has shown no global efficiency in MM patients without indication of their subgroup origin and their CCND1 expression [45]. However, since it is efficient (in combination) in patients with tumors overexpressing CCND1 such as mantle cell lymphoma or $\mathrm{HR}^{+}$breast cancer, it might be of interest for patients with $\mathrm{t}(11 ; 14)$ without $\mathrm{Rb}$ deficiency $[46,47]$. Concerning TP53, we previously described p53 independent drugs, which were efficient whatever TP53 status, such as PRIMA-1 ${ }^{\text {Met }}$ that targets glutathione or BH3 mimetics that target anti-apoptotic proteins $[6,11]$. We also reported that loss of p53 function favors measles virus replication and cell death in myeloma cells 


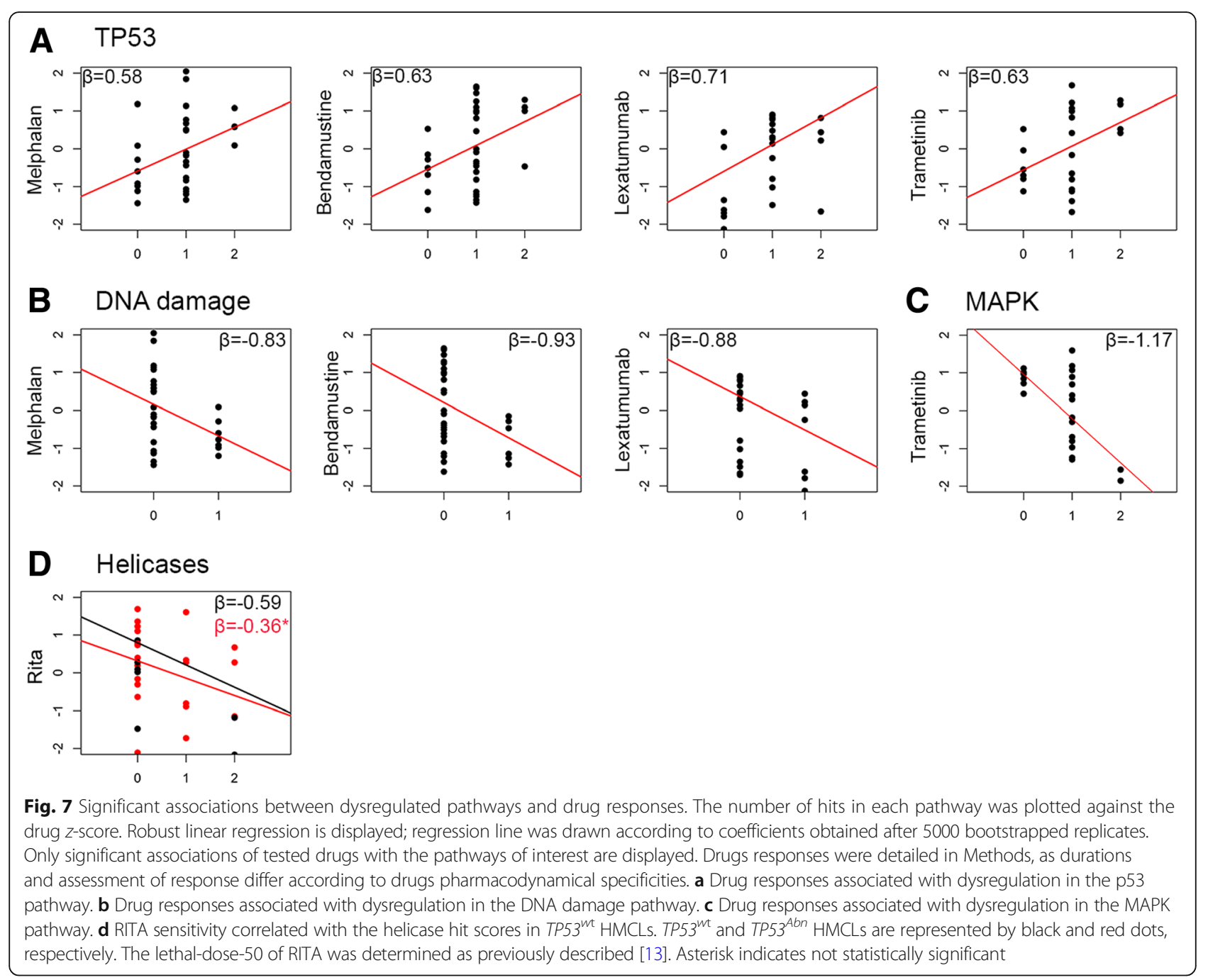

[48]. FAM46C was recently been shown to encode for a non-canonical poly(A) polymerase and its over expression in MM cells induced cell death [49]. FAM46C is a type I IFN-stimulated gene, and it might modulate virus replication such as the yellow fever virus (YFV) and the Venezuelan equine encephalitis virus (VEEV) [50]. Of note, anti-viral type I IFN pathway appeared highly impaired, suggesting defects in infection defense that might be exploited using oncolytic viruses such as measles virus $[48,51]$.

Concerning mutations with gain of function such as $R A S$ mutations, we showed that sensitivity of 27 HMCLs to MEK1/2 inhibitor trametinib was associated to $R A S$ mutations (70\% of "RAS only" mutated HMCLs were sensitive), but not to FGFR3 (none sensitive HMCL out of three with "FGFR3 only" mutation). Concerning $B R A F$, four HMCLs out of five with BRAF mutation (and with NRAS mutations for two of them) were sensitive but the low number of "BRAF only" mutated HMCLs prevented definitive conclusions. Although all
HMCLs without hit in RAS/BRAF/FGFR3 genes were resistant to trametinib, all HMCLs with NRAS mutation were not sensitive since four NRAS mutated HMCLs were resistant. These data collectively suggest that mutation in $R A S / B R A F$ genes is required but not sufficient for eliciting response to trametinib. The $B R A F / R A S$ impact will be assessed in an ongoing clinical trial (NCT03091257) evaluating dabrafenib and/or trametinib in patients with relapsed and/or refractory multiple myeloma patients according to their BRAF/RAS mutation.

The high percentage of altered genes in DNA/chromatin repair/regulation, Fanconi pathway, and chromatin/ DNA modification might be related to the frequency in relapsing patients [32]. Because of the lack of specific drugs, we could not directly assess the functionality/vulnerability of these pathways, which require a deep investigation. Of note, mutations in Fanconi genes were recently reported in patients at relapse, suggesting that drug escape might involve this pathway. HCLMs exhibiting such "BRCAness" will be a good model for assessing 
efficiency of drugs like USP1 and/or PARP inhibitors $[23,31,52]$.

On the other hand, no major alteration was found in apoptosis pathway, either extrinsic/intrinsic or executive, showing that resistance to cell death was rather upstream of the mitochondria. In good agreement with the low number of alterations in apoptosis pathway, HMCLs were highly primed for death as shown by their $\mathrm{BH} 3$ profiling and their high response rate to $\mathrm{BH} 3$ mimetics [11, 53] (Additional file 1: Table S2). Considering the huge difference between cell responses to DNA damaging drugs and $\mathrm{BH} 3$ mimetics, loss of response was not on the mitochondrial side, and $\mathrm{BH} 3$ mimetics appear thus of major interest to target MM cells whatever their genomic alterations or responses to classical myeloma drugs.

\section{Conclusions}

In summary, WES suggests that HMCLs harbor enriched mutations and defects in cell cycle, p53, recombination/ DNA repair, $\mathrm{NFkB}$, and epigenetic genes. Importantly, some very early pathogenic events such as IgH translocations and MAPK pathway mutants are stable over time and are not enriched by in vitro long-term culture, thus making HMCLs a reliable drug screening model for refractory patients at diagnosis or relapse. What is more, detection at diagnosis of mutations/deletions in genes associated with progression and HMCLs (i.e., CDKN2C, FAM46C, TRAF3, PRKD2) might identify particularly aggressive sub-clones warranting adapted treatment strategies and surveillance. WES results suggest that in addition to target apoptosis using $\mathrm{BH} 3$ mimetics and the antiviral deficiency using oncolytic viruses, targeting DNA damage, recombination/DNA repair, and epigenetic modifiers should be further investigated and might offer significant options for high-risk and refractory patients, including extramedullary diseases.

\section{Additional files}

Additional file 1: Additionnal Analyses and Plots. (DOCX 17568 kb)

Additional file 2: MAF File for Sequence variants. (XLSX $16253 \mathrm{~kb}$ )

Additional file 3: Lollipop plots of the main sequence variants. (PDF $2025 \mathrm{~kb}$ )

\section{Abbreviations}

DMM: Multiple myeloma at diagnosis; HMCL: Human myeloma cell lines; PCL: Plasma cell leukemia; PPCL: Primary PCL; RMM: Relapsing multiple myeloma; SNP: Single nucleotide polymorphism; sPCL: Secondary PCL; WES: Whole-exon sequencing

\section{Acknowledgements}

We are grateful to the BiRD bioinformatics facility for providing support and computing resources. We thank Antoine Lamothe and Jeanne Chauvat for performing MTT assays.

\section{Funding}

This work was supported by grant from FFRMG, Action Cancer 44, DHU Oncogreffe, Ligue Grand Ouest contre le Cancer, SIRIC ILIAD (INCa-DGOSInserm_12558), and L'Héma-NexT (i-site NexT). BT was supported by INSERM (poste d'accueil) and Fondation ARC. AG was supported by the SyMeTRIC project, funded by the Région Pays de la Loire Connect Talent research call.

\section{Availability of data and materials}

All data and materials are provided in the Additional files; Mutant Annotation File (MAF) is hosted as a separate file at Journal of Hematology \& Oncology. Raw data will be submitted at COSMIC website (https://cancer.sanger.ac.uk/ cosmic) after manuscript approval for publication, due to COSMIC restrictions. Gene Expression Profiling data are available at ArrayExpress public database (https://www.ebi.ac.uk/arrayexpress/) under accession numbers E-TABM-937 and E-TABM-1088.

\section{Authors' contributions}

BT designed the project, performed bioinformatics analyses, and wrote the paper. AG participated in the bioinformatics analyses. AMA, GD, PGB, SM, DC, and EM performed experiments. SLG, PM, and MA participated in the design of the project. CPD designed the project and wrote the paper. All authors read and approved the final manuscript.

\section{Ethics approval}

Not applicable

\section{Consent for publication}

Not applicable; all data and figures are ours.

\section{Competing interests}

The authors declare that they have no competing interests.

\section{Publisher's Note}

Springer Nature remains neutral with regard to jurisdictional claims in published maps and institutional affiliations.

\section{Author details}

${ }^{1}$ CRCINA, INSERM, CNRS, Université d'Angers, Université de Nantes, Nantes, France. ${ }^{2}$ Service d'Hématologie Clinique, Unité d'Investigation Clinique, CHU, Nantes, France. ${ }^{3}$ Bird Platform, Inserm 1087, Nantes, France. ${ }^{4}$ Myelomax SAS, Nantes, France.

Received: 12 July 2018 Accepted: 20 November 2018

Published online: 13 December 2018

\section{References}

1. Moreaux J, Klein B, Bataille R, Descamps G, Maïga S, Hose D, et al. A highrisk signature for patients with multiple myeloma established from the molecular classification of human myeloma cell lines. Haematologica. 2011; 96(4):574-82.

2. Manier S, Salem KZ, Park J, Landau DA, Getz G, Ghobrial IM. Genomic complexity of multiple myeloma and its clinical implications. Nat Rev Clin Oncol. 2017;14(2):100-13.

3. Sonneveld P, Avet-Loiseau H, Lonial S, Usmani S, Siegel D, Anderson KC, et al. Treatment of multiple myeloma with high-risk cytogenetics: a consensus of the International Myeloma Working Group. Blood. 2016; 127(24):2955-62.

4. Moreau P, Cavo M, Sonneveld P, Rosinol L, Attal M, Pezzi A, et al. Combination of international scoring system 3 , high lactate dehydrogenase, and $t(4 ; 14)$ and/or del(17p) identifies patients with multiple myeloma (MM) treated with front-line autologous stem-cell transplantation at high risk of early MM progression-related death. J Clin Oncol. 2014;32(20):2173-80.

5. Sonneveld P, De Wit E, Moreau P. How have evolutions in strategies for the treatment of relapsed/refractory multiple myeloma translated into improved outcomes for patients? Crit Rev Oncol Hematol. 2017;112:153-70.

6. Tessoulin B, Descamps G, Moreau P, Maïga S, Lodé L, Godon C, et al. PRIMA1 Met induces myeloma cell death independent of p53 by impairing the GSH/ROS balance. Blood. 2014;124(10):1626-36.

7. Maïga S, Brosseau C, Descamps G, Dousset C, Gomez-Bougie P, Chiron D, et al. A simple flow cytometry-based barcode for routine authentication of 
multiple myeloma and mantle cell lymphoma cell lines. Cytometry A. 2015; 87(4):285-8.

8. Zhan F, Huang Y, Colla S, Stewart JP, Hanamura I, Gupta S, et al. The molecular classification of multiple myeloma. Blood. 2006;108(6):2020-8.

9. Surget $S$, Chiron D, Gomez-Bougie $P$, Descamps G, Ménoret E, Bataille R, et al. Cell death via DR5, but not DR4, is regulated by p53 in myeloma cells. Cancer Res. 2012;72(17):4562-73.

10. Bodet L, Gomez-Bougie P, Touzeau C, Dousset C, Descamps G, Maïga S, et al. ABT-737 is highly effective against molecular subgroups of multiple myeloma. Blood. 2011;118(14):3901-10.

11. Touzeau C, Dousset C, Le Gouill S, Sampath D, Leverson JD, Souers AJ, et al. The BCl-2 specific BH3 mimetic ABT-199: a promising targeted therapy for $\mathrm{t}(11 ; 14)$ multiple myeloma. Leukemia. 2014;28(1):210-2.

12. Surget S, Lemieux-Blanchard E, Maïga S, Descamps G, Le Gouill S, Moreau P, et al. Bendamustine and melphalan kill myeloma cells similarly through reactive oxygen species production and activation of the $\mathrm{p} 53$ pathway and do not overcome resistance to each other. Leuk Lymphoma. 2014;55(9):2165-73.

13. Surget $S$, Descamps $G$, Brosseau C, Normant V, Maïga S, Gomez-Bougie P, et al. RITA (reactivating p53 and inducing tumor apoptosis) is efficient against TP53 abnormal myeloma cells independently of the p53 pathway. BMC Cancer. 2014;14:437.

14. Kervoëlen C, Ménoret E, Gomez-Bougie P, Bataille R, Godon C, Marionneau-Lambot $\mathrm{S}$, et al. Dexamethasone-induced cell death is restricted to specific molecular subgroups of multiple myeloma. Oncotarget. 2015;6(29):26922-34.

15. Gerstung M, Pellagatti A, Malcovati L, Giagounidis A, Porta MGD, Jädersten M, et al. Combining gene mutation with gene expression data improves outcome prediction in myelodysplastic syndromes. Nat Commun. 2015;6:5901.

16. Yu G, He Q-Y. ReactomePA: an R/Bioconductor package for reactome pathway analysis and visualization. Mol Biosyst. 2016;12(2):477-9.

17. Yu G, Wang L-G, Han Y, He Q-Y. clusterProfiler: an R package for comparing biological themes among gene clusters. OMICS. 2012;16(5):284-7.

18. Mayakonda A, Lin DC, Assenov Y, Plass C, Koeffler HP. Maftools: efficient and comprehensive analysis of somatic variants in cancer. Genome Res. 2018; 28(11):1747-56. https://doi.org/10.1101/gr.239244.118

19. Zheng X, Levine D, Shen J, Gogarten SM, Laurie C, Weir BS. A highperformance computing toolset for relatedness and principal component analysis of SNP data. Bioinformatics. 2012;28(24):3326-8.

20. Walker BA, Boyle EM, Wardell CP, Murison A, Begum DB, Dahir NM, et al. Mutational spectrum, copy number changes, and outcome: results of a sequencing study of patients with newly diagnosed myeloma. J Clin Oncol. 2015;33(33):3911-20.

21. Chapman MA, Lawrence MS, Keats JJ, Cibulskis K, Sougnez C, Schinzel AC, et al. Initial genome sequencing and analysis of multiple myeloma. Nature. 2011;471(7339):467-72.

22. Lohr JG, Stojanov P, Carter SL, Cruz-Gordillo P, Lawrence MS, Auclair D, et al. Widespread genetic heterogeneity in multiple myeloma: implications for targeted therapy. Cancer Cell. 2014;25(1):91-101.

23. Chavan SS, He J, Tytarenko R, Deshpande S, Patel P, Bailey M, et al. Bi-allelic inactivation is more prevalent at relapse in multiple myeloma, identifying RB1 as an independent prognostic marker. Blood Cancer J. 2017;7(2):e535.

24. Weinhold N, Ashby C, Rasche L, Chavan SS, Stein C, Stephens OW, et al. Clonal selection and double-hit events involving tumor suppressor genes underlie relapse in myeloma. Blood. 2016;128(13):1735-44.

25. Barbieri M, Manzoni M, Fabris S, Ciceri G, Todoerti K, Simeon V, et al. Compendium of FAM46C gene mutations in plasma cell dyscrasias. $\mathrm{Br} \mathrm{J}$ Haematol. 2016;174(4):642-5.

26. Cifola I, Lionetti M, Pinatel E, Todoerti K, Mangano E, Pietrelli A, et al. Wholeexome sequencing of primary plasma cell leukemia discloses heterogeneous mutational patterns. Oncotarget. 2015:6(19):17543-58.

27. Lionetti M, Barbieri M, Todoerti K, Agnelli L, Marzorati S, Fabris S, et al. Molecular spectrum of BRAF, NRAS and KRAS gene mutations in plasma cell dyscrasias: implication for MEK-ERK pathway activation. Oncotarget. 2015; 6(27):24205-17.

28. Lionetti M, Barbieri M, Manzoni M, Fabris S, Bandini C, Todoerti K, et al. Molecular spectrum of TP53 mutations in plasma cell dyscrasias by next generation sequencing: an Italian cohort study and overview of the literature. Oncotarget. 2016;7(16):21353-61.

29. Jurczyszyn A, Castillo JJ, Avivi I, Czepiel J, Davila J, Vij R, et al. Secondary plasma cell leukemia: a multicenter retrospective study of 101 patients. Leuk Lymphoma. 2018;0(0):1-6.
30. Lagana A, Melnekoff D, Beno I, Leshchenko V, Perumal D, Keats JJ, et al. Clonal evolution in newly diagnosed multiple myeloma patients: a followup study from the Mmrf Commpass Genomics Project. Blood. 2017; 130(Suppl 1):325.

31. Lehners N, Toprak UH, Xu J, Paramasivam N, Hübschmann D, Fröhlich M, et al. Comprehensive genomic characterization of refractory multiple myeloma reveals a complex mutational and structural landscape associated with drug resistance. Blood. 2017;130(Suppl 1):266.

32. Pawlyn C, Kaiser MF, Heuck C, Melchor L, Wardell CP, Murison A, et al. The spectrum and clinical impact of epigenetic modifier mutations in myeloma. Clin Cancer Res. 2016;22(23):5783-94.

33. Tessoulin B, Eveillard M, Lok A, Chiron D, Moreau P, Amiot M, Moreau-Aubry A, Le Gouill S, Pellat-Deceunynck C. p53 dysregulation in B-cell malignancies: More than a single gene in the pathway to hell. Blood Rev. 2017;31(4):251-59. https://doi.org/10.1016/j.blre.2017.03.001

34. Tao Z-F, Hasvold L, Wang L, Wang X, Petros AM, Park CH, et al. Discovery of a potent and selective BCL-XL inhibitor with in vivo activity. ACS Med Chem Lett. 2014;5(10):1088-93.

35. Leverson JD, Zhang H, Chen J, Tahir SK, Phillips DC, Xue J, et al. Potent and selective small-molecule MCL-1 inhibitors demonstrate on-target cancer cell killing activity as single agents and in combination with ABT-263 (navitoclax). Cell Death Dis. 2015;6:e1590.

36. Finn RS, Dering J, Conklin D, Kalous O, Cohen DJ, Desai AJ, et al. PD 0332991, a selective cyclin D kinase 4/6 inhibitor, preferentially inhibits proliferation of luminal estrogen receptor-positive human breast cancer cell lines in vitro. Breast Cancer Res. 2009;11(5):R77.

37. Annunziata CM, Davis RE, Demchenko Y, Bellamy W, Gabrea A, Zhan F, et al. Frequent engagement of the classical and alternative NF-kB pathways by diverse genetic abnormalities in multiple myeloma. Cancer Cell. 2007;12(2):115-30.

38. Nieves-Neira W, Rivera MI, Kohlhagen G, Hursey ML, Pourquier P, Sausville EA, et al. DNA protein cross-links produced by NSC 652287, a novel thiophene derivative active against human renal cancer cells. Mol Pharmacol. 1999;56(3):478-84.

39. Lee HC, Wang H, Baladandayuthapani V, Lin H, He J, Jones RJ, et al. RNA polymerase I inhibition with CX-5461 as a novel therapeutic strategy to target MYC in multiple myeloma. Br J Haematol. 2017;177(1):80-94.

40. Bolli N, Li Y, Sathiaseelan V, Raine K, Jones D, Ganly P, et al. A DNA targetenrichment approach to detect mutations, copy number changes and immunoglobulin translocations in multiple myeloma. Blood Cancer J. 2016; 6(9):e467.

41. Drexler HG, Dirks WG, MacLeod RA. False human hematopoietic cell lines: cross-contaminations and misinterpretations. Leukemia. 1999;13(10):1601-7.

42. Drexler HG, Dirks WG, MacLeod RA, Uphoff CC. False and mycoplasmacontaminated leukemia-lymphoma cell lines: time for a reappraisal. Int J Cancer. 2017;140(5):1209-214.

43. Hanahan D, Weinberg RA. Hallmarks of cancer: the next generation. Cell. 2011;144(5):646-74.

44. Klein ME, Kovatcheva M, Davis LE, Tap WD, Koff A. CDK4/6 inhibitors: the mechanism of action may not be as simple as once thought. Cancer Cell. 2018;34(1):9-20.

45. Niesvizky R, Badros AZ, Costa L, Ely SA, Singhal SB, Stadtmauer EA, et al. Phase 1/2 study of cyclin-dependent kinase (CDK)4/6 inhibitor palbociclib (PD-0332991) with bortezomib and dexamethasone in relapsed/refractory multiple myeloma. Leuk Lymphoma. 2015;56(12):3320-8.

46. Leonard JP, LaCasce AS, Smith MR, Noy A, Chirieac LR, Rodig SJ, et al. Selective CDK4/6 inhibition with tumor responses by PD0332991 in patients with mantle cell lymphoma. Blood. 2012;119(20):4597-607.

47. Kwapisz D. Cyclin-dependent kinase $4 / 6$ inhibitors in hormone receptorpositive early breast cancer: preliminary results and ongoing studies. Breast Cancer. 2018:25(5):506-16.

48. Lok A, Descamps G, Tessoulin B, Chiron D, Eveillard M, Godon C, Le Bris Y, Vabret A, Bellanger C, Maillet L, Barillé-Nion S, Gregoire M, Fonteneau J-F, Le Gouill S, Moreau P, Tangy F, Amiot M, Moreau-Aubry A, Pellat-Deceunynck C. p53 regulates CD46 expression and Measles virus infection in myeloma cells. Blood Advances. In press.

49. Mroczek S, Chlebowska J, Kuliński TM, Gewartowska O, Gruchota J, Cysewski $D$, et al. The non-canonical poly(A) polymerase FAM46C acts as an oncosuppressor in multiple myeloma. Nat Commun. 2017;8(1):619.

50. Schoggins JW, Wilson SJ, Panis M, Murphy MY, Jones $C T$, Bieniasz P, et al. A diverse range of gene products are effectors of the type I interferon antiviral response. Nature. 2011;472(7344):481-5. 
51. Dispenzieri A, Tong C, LaPlant B, Lacy MQ, Laumann K, Dingli D, et al. Phase I trial of systemic administration of Edmonston strain of measles virus genetically engineered to express the sodium iodide symporter in patients with recurrent or refractory multiple myeloma. Leukemia. 2017;31 (12):2791-8.

52. Christofferson A, Nasser S, Aldrich J, Penaherrera D, Legendre C, Benard B, et al. Integrative analysis of the genomic landscape underlying multiple myeloma at diagnosis: an Mmrf Commpass Analysis. Blood. 2017;130(Suppl 1):326.

53. Kotschy A, Szlavik Z, Murray J, Davidson J, Maragno AL, Le Toumelin-Braizat $\mathrm{G}$, et al. The MCL1 inhibitor $\mathbf{S 6 3 8 4 5}$ is tolerable and effective in diverse cancer models. Nature. 2016;538(7626):477-82.

Ready to submit your research? Choose BMC and benefit from:

- fast, convenient online submission

- thorough peer review by experienced researchers in your field

- rapid publication on acceptance

- support for research data, including large and complex data types

- gold Open Access which fosters wider collaboration and increased citations

- maximum visibility for your research: over $100 \mathrm{M}$ website views per year

At $\mathrm{BMC}$, research is always in progress.

Learn more biomedcentral.com/submissions 